\title{
Nekton community of the Scotia Sea as sampled by the RMT 25 during austral summer
}

\author{
Uwe Piatkowski $^{1}$, Paul G. Rodhouse ${ }^{2}$, Martin G. White ${ }^{2}$, Douglas G. Bone ${ }^{2}$, \\ Carolyn Symon ${ }^{2}$
}

\author{
${ }^{1}$ Institut für Meereskunde, Universität Kiel, Düsternbrooker Weg 20, D-24105 Kiel, Germany \\ ${ }^{2}$ British Antarctic Survey, Natural Environment Research Council, High Cross, Madingley Road, Cambridge CB3 0ET, \\ United Kingdom
}

\begin{abstract}
The nekton community was sampled by a Rectangular Midwater Trawl (RMT 25) over the upper $1000 \mathrm{~m}$ of the Scotia Sea during January 1991. A total of 81 nekton and micronekton species were collected from 2 sites, one in the oceanic western Scotia Sea (Stn 1) and the other on the northwestern slope of the South Georgia shelf (Stn 2). Species composition, abundance, biomass and day/night vertical distribution were investigated. Crustaceans were the most important group in terms of species numbers (28 species) followed by mesopelagic fish (24), molluscs (15) and coelenterates (11). Species diversity increased with depth and was higher at Stn 2 (76 species) than at Stn 1 (62 species). Biomass in the upper $1000 \mathrm{~m}$ was considerably higher at Stn $1\left(94.6 \mathrm{~g}\right.$ wet wt $\mathrm{m}^{-2}$ during daytime, $87 \mathrm{~g}$ wet wt $\mathrm{m}^{-2}$ during night) than at $\operatorname{Stn} 2$ (10.2 and $23.7 \mathrm{~g}$ wet $\mathrm{wt} \mathrm{m}^{-2}$, respectively), mostly due to dense concentrations of the tunicate Salpa thompsoni (41.6 g wet wt $\mathrm{m}^{-2}$ during night). The other main contributors to the high biomass at Stn 1 were coelenterates ( $28.3 \mathrm{~g}$ wet wt $\mathrm{m}^{-2}$ during night) and mesopelagic fish ( $4.9 \mathrm{~g}$ wet wt $\mathrm{m}^{-2}$ during night). Euphausiids (Euphausia triacantha and E. superba) accounted for $1.5 \mathrm{~g}$ wet wt $\mathrm{m}^{-2}$ at $\operatorname{Stn} 2$ during night, with $E$. triacantha the more important of the two $\left(1.4 \mathrm{~g}\right.$ wet wt $\left.\mathrm{m}^{-2}\right)$. Except for Bathylagus antarcticus all common mesopelagic fishes showed a marked diurnal vertical migration (i.e. Electrona antarctica, Gymnoscopelus braueri, Krefftichthys anderssoni, Protomyctophum bolini). During daylight they stayed in the core of the Circumpolar Deep Water (CDW; 400 to $800 \mathrm{~m}$ ) and at night they were mainly distributed in the Antarctic Surface Water (ASW; 0 to $400 \mathrm{~m}$ ). Other species with pronounced vertical migration were the hydromedusa Calycopsis borchgrevinki, the squid Brachioteuthis ?picta, and the euphausiid Euphausia triacantha. The scyphomedusae Atolla wyvillei and Periphylla periphylla and the crustaceans Cyphocaris richardi, Gigantocypris mulleri and Pasiphaea scotiae did not appear to migrate and remained concentrated in the CDW. Spatial variability was analysed by multivariate data analyses (clustering techniques) and related to hydrography. Four main groups, characterised by different nekton communities, were derived: (1) a lower mesopelagic nekton community from the deeper layers of the CDW, apparent at both stations, (2) an upper mesopelagic nekton community from the core of the CDW, apparent at both stations, (3) an epipelagic nekton community from the ASW over the South Georgia slope (Stn 2) and finally (4) an epipelagic nekton community from the ASW of the oceanic Scotia Sea (Stn 1). The performance of the midwater trawl is discussed as it has a substantial impact on the catchability of the nekton. The presented data provide new information on the structure and spatial variability of Antarctic nekton communities and emphasise the geographical and vertical discontinuities between communities.
\end{abstract}

KEY WORDS: Nekton community · Southern Ocean · Pelagic ecosystem · Antarctic food chain · RMT 25

\section{INTRODUCTION}

It has been suggested that the Southern Ocean can no longer be considered a single ecosystem (Clarke 1985, Hempel 1985). In fact there is a pronounced hydrographic and biogeographic latitudinal zonation separating the region into 3 large-scale subsystems: the ice free oceanic 'West Wind Drift' (hereinafter referred to as Antarctic Circumpolar Current, ACC) extending from the Subtropical Front in the north to the northern limit of the pack ice, the 'East Wind Drift' with seasonal pack ice cover, and the shelf water zone immediately around the Antarctic landmass with almost permanent ice cover (Hempel 1985). The nekton samples analysed in the present study were all obtained from the Scotia Sea (Fig. 1), which is charac- 


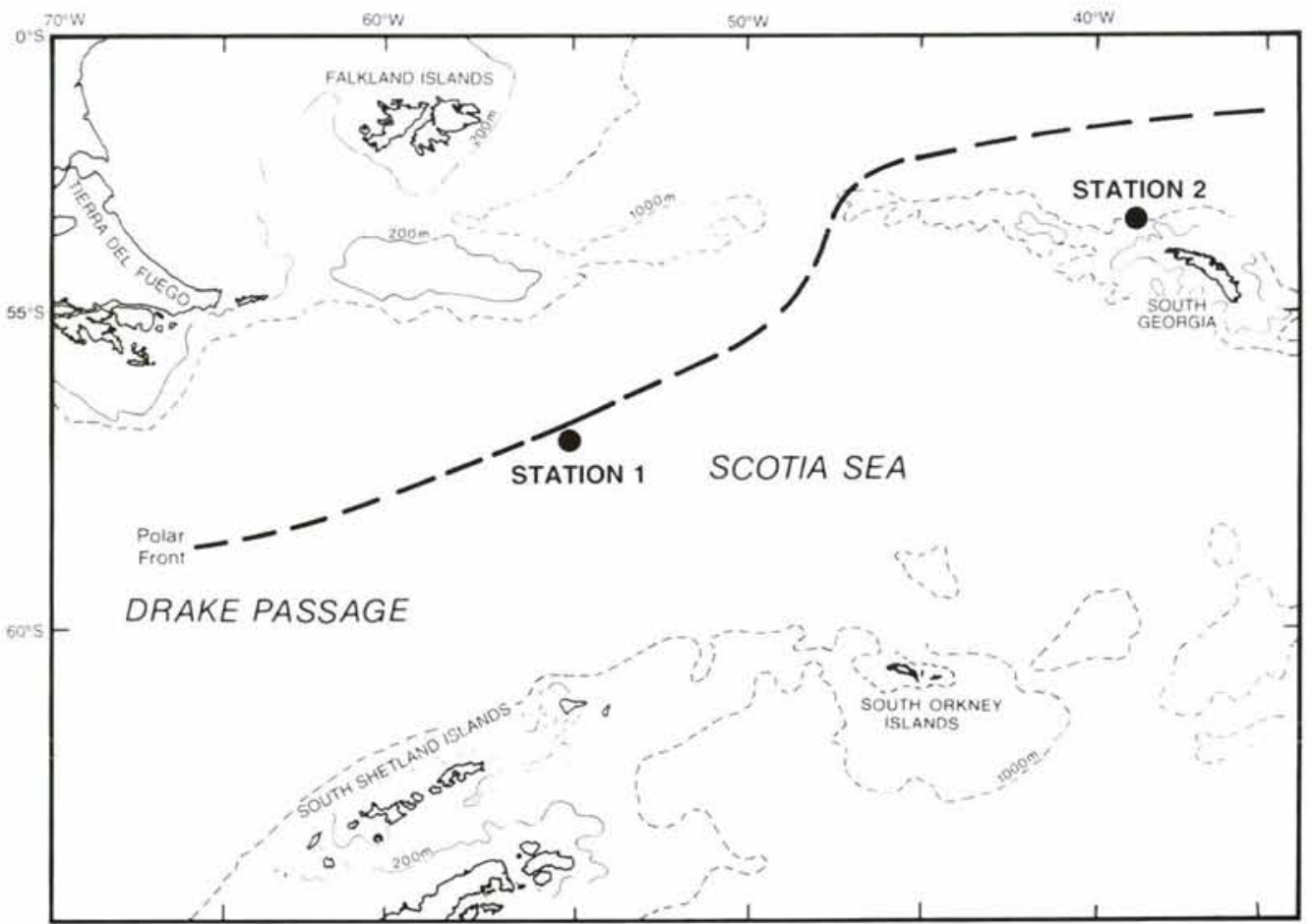

Fig. 1. Atlantic sector of the Southern Ocean showing the Polar Front relative to $\operatorname{Stn} 1$ ( 13 to 15 January 1991) and Stn 2 (28 to 30 January 1991)

terised by a high zooplankton diversity (copepods, small euphausiids and salps predominating; Mackintosh 1934, Foxton 1966, Hempel 1985), and occurs in the most northerly of the 3 sectors.

Despite intensive investigations of the zooplankton and macrozooplankton communities of the Atlantic sector of the Southern Ocean (e.g. Hopkins 1985a, Atkinson \& Peck 1988, Boysen-Ennen \& Piatkowski 1988, Hopkins \& Torres 1988, Piatkowski 1989a, b, Siegel \& Piatkowski 1990, Siegel et al. 1992, Hopkins et al. 1993) the ecology of the micronekton or the nekton communities has received relatively little attention (Lancraft et al. 1989, 1991). Of all known species of Antarctic fish, $25 \%$ live in the meso- and bathypelagic regions of the Southern Ocean (Kock 1992) and form a substantial part of the nekton community. Although the general biogeography of these species has been described (Hulley 1981, McGinnis 1982, Gon \& Heemstra 1990) their detailed ecology is largely undescribed. In recent years the most abundant group, the myctophids, which occur in dense concentrations in the Scotia Sea, have begun to be exploited commercially (Filin et al. 1991, Kozlov et al. 1991). They are also important in the diet of a number of vertebrate predators, particularly king penguins (e.g. Adams \& Klages 1987, Adams \& Brown 1989, Cherel \& Ridoux 1992) and this highlights their importance in the Antarctic food web. The nekton community also contains large populations of coelenterates (Larson 1986, Larson \& Harbison 1990) and populations of large pelagic shrimps for which there are few ecological data (Clarke \& Holmes 1987, Iwasaki \& Nemoto 1987). Squid are also important components of the nekton community, especially the ommastrephid Martialia hyadesi (Rodhouse \& Yeatman 1990, Rodhouse 1991). This forms the principal prey item for a number of vertebrate predators (Croxall \& Lishman 1987, Rodhouse et al. 1987, 1990, 1992a, Rodhouse \& Prince 1993), which are together estimated to consume some 340000 t of $M$. hyadesi per year in the Scotia Sea (Rodhouse et al. 1993). In turn, $M$. hyadesi feeds on other members of the nekton community, for example myctophids and euphausiids (Rodhouse et al. 1992b). In addition to the myctophids, $M$. hyadesi is also considered to be a resource with potential for future commercial exploitation (Rodhouse 1990). In the absence of any true epipelagic families of fish in the Antarctic (Kock 1987, 1992) the ommastrephid squids of the Scotia Sea appear to fill the niche occupied elsewhere in the world's oceans by pelagic fish such as carangids, clupeids and scombrids.

Thus, any model of the Southern Ocean pelagic ecosystem will be incomplete without a consideration of the composition, abundance and standing stock of the nekton. This paper describes the nekton community of the Scotia Sea, defining community according to Mills (1969) as 'a group of organisms occurring in a particular environment, presumably interacting with each other and with the environment, and separable by means of ecological survey from other groups'. 
This study examines the nekton community of the upper $1000 \mathrm{~m}$ at 2 sites in the Scotia Sea using hauls made with an opening/closing midwater trawl. The first of these was oceanic (Stn 1), while the other occurred on the northwestern slope of the South Georgia shelf (Stn 2). Both stations were located in the northern Scotia Sea close to the main path of the ACC. The ACC is the major oceanographic feature of the Southern Ocean and is an extensive, eastward flowing circumpolar current (e.g. Whitworth 1983, Hellmer et al. 1985, Stramma \& Peterson 1990), responsible for the circumpolar distribution of zooplankton and micronekton species. It flows into the Scotia Sea through the Drake Passage before being deflected to the north of South Georgia. The upper waters of the ACC comprise Antarctic Surface Water (ASW) and Subantarctic Surface Water separated by a band of Antarctic Intermediate Water (AIW) and underlain by a thick band of Circumpolar Deep Water (CDW). The AIW is limited to the north by the Subantarctic Front (SAF) and to the south by the Polar Front (PF). Both sample sites occurred to the south of the PF, a region considered to be highly productive (Ainley \& DeMaster 1990).

We report on the spatial distribution patterns of the nekton species at these 2 sites and compare the stations using clustering techniques. Detailed analyses on the biomass spectra of the nekton community and their implications for the upper pelagic food web in the Antarctic are described in the companion article by Rodhouse et al. (1994).

\section{MATERIALS AND METHODS}

Sampling. Nekton samples were collected with an opening/closing rectangular midwater trawl with a design aperture of $25 \mathrm{~m}^{2}$ (RMT 25) according to the design principle described by Baker et al. (1973). The trawl consists of 2 nets, opened and closed using release gear connected to an IOS type acoustic net monitor (Harris 1969). The release gear had 4 operational jaws allowing the nets to be rigged so that opening the second net was independent of closing the first. This enabled separate layers to be sampled. The design mouth-angle of the net is $45^{\circ}$ and, for the purpose of calculating water volume sampled, it was assumed that this angle was maintained at the towing speed of 3 knots. The nets were built in 3 sections of reducing mesh size: 19,10 and $5 \mathrm{~mm}$, measured along 1 side of the square. All the meshes were of woven, knotless construction. Actual panel sizes are shown in Fig. 2 together with the design mouth area for each mesh section. The cod-ends employed on these nets are the standard type used with the RMT $1+8$ system (Baker et al. 1973). (a)

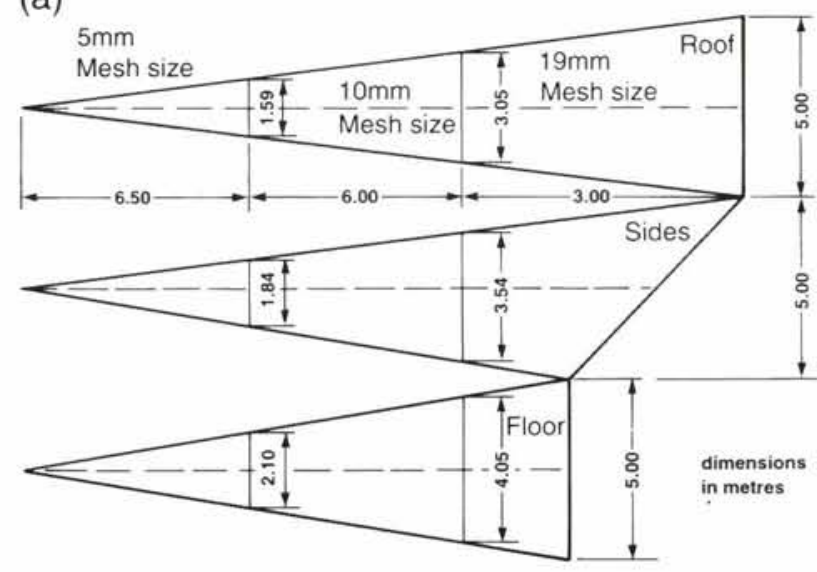

(b)

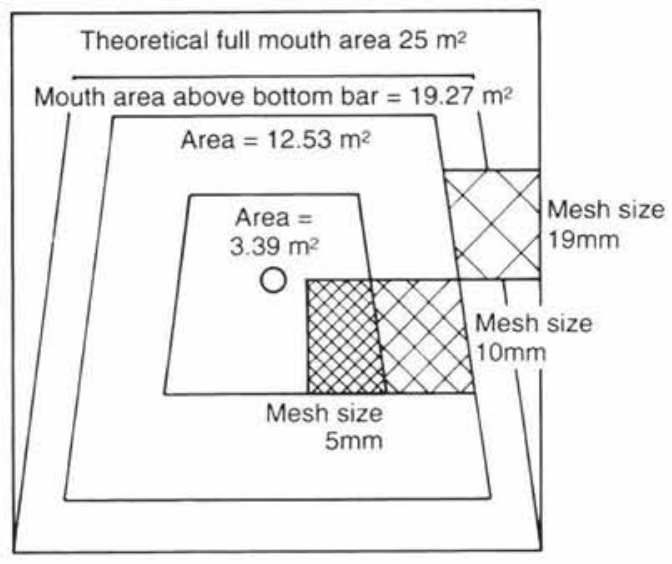

Fig. 2. The British Antarctic Survey's RMT 25. (a) Size and shape of the 3 panels of netting. (b) Mouth areas for each mesh size

A total of 20 RMT 25 samples were collected in the austral summer at Stn 1 in the oceanic Scotia Sea (bottom depth $4100 \mathrm{~m} ; 13$ to 15 January 1991), and at Stn 2 on the northwestern slope of South Georgia (bottom depth $1500 \mathrm{~m} ; 28$ to 30 January 1991) (Fig. 1). At Stn 1 all net hauls fell within a rectangle ranging from $57^{\circ} 03.8^{\prime} \mathrm{S}$ to $57^{\circ} 08.7^{\prime} \mathrm{S}$, and from $55^{\circ} 08.6^{\prime} \mathrm{W}$ to $55^{\circ} 25.9^{\prime} \mathrm{W}$; at $\mathrm{Stn} 2$ all net hauls fell within a rectangle ranging from $53^{\circ} 22.5^{\prime} \mathrm{S}$ to $53^{\circ} 39.1^{\prime} \mathrm{S}$, and from $38^{\circ} 31.9^{\prime} \mathrm{W}$ to $38^{\circ} 55.7^{\prime} \mathrm{W}$. Each $200 \mathrm{~m}$ layer from the surface to $1000 \mathrm{~m}$ was sampled twice at each station, once in daylight and once at night, as defined by sunrise and sunset. The RMT 25 was deployed in downward oblique hauls. While it was fishing the net was lowered slowly and continuously through the target layer. The duration of a standard haul within one layer was $2 \mathrm{~h}$ but in some cases this was extended for up to $1 \mathrm{~h}$. The mean volume filtered per $2 \mathrm{~h}$ haul was 260000 $\pm 48000 \mathrm{~m}^{3}$. Flowmeter data were used to correct all samples to a standard volume of $10000 \mathrm{~m}^{3}$. 
Between the RMT 25 hauls temperature and salinity profiles were obtained for the top $1000 \mathrm{~m}$ using a Neil Brown Mark III CTD.

Treatment of samples. The total volume of each net sample was measured and in the case of large samples, sub-samples were taken prior to sorting. Nekton organisms, arbitrarily defined as metazoans $>10 \mathrm{~mm}$ in body size, were identified to the lowest posssible taxonomic unit. Siphonophores, copepods, small polychaetes, amphipods and chaetognaths, which occurred regularly in the samples, were considered too small to be sampled quantitatively by the RMT 25 and were discarded after sorting. Ctenophores were also excluded from further calculations because they could not be collected as whole specimens. The total number of each nektonic species was recorded and the total volume of each species determined by displacement. Biomass was expressed in standing stock $(\mathrm{g}$ wet $\mathrm{wt}$ $\mathrm{m}^{-2}, \mathrm{mg}$ wet wt $\mathrm{m}^{-2}$ ) assuming $1 \mathrm{~g}$ approximately equals $1 \mathrm{ml}$ (displacement volume). Species relative abundance was expressed in individuals per $10000 \mathrm{~m}^{3}$ water volume filtered (ind. $10^{-4} \mathrm{~m}^{-3}$ ).

Treatment of data. Agglomerative hierarchical cluster analysis was used to differentiate and group the 20 samples from the 2 stations. The analyses followed a multi-step strategy for the study of multi-species distribution patterns (Field et al. 1982). In order to avoid overemphasizing rare and random occurrences the data set was reduced to the 58 taxa that occurred in at least 3 samples. Data were arranged in an $(n \times m)$ matrix with $n$ representing the 20 samples and $m$ representing the relative abundances (ind. $10^{-4} \mathrm{~m}^{-3}$ ) of the 58 nekton taxa. All species abundances were $\log _{10^{-}}$ transformed to smooth the large abundance values of a few species. Similarity between sample pairs was expressed by the Bray-Curtis Index; this index groups the samples according to both presence and abundance of species. This is the most appropriate method for marine ecological data sets (Field et al. 1982). The clustering of samples followed the Complete Linkage Method. Cluster analyses and calculations of ecological indices were carried out using the software package COMM adapted for personal computers (Piepenburg \& Piatkowski 1992). More information on the various clustering procedures and their effects on classification results is compiled by Romesburg (1984).

\section{RESULTS}

\section{Hydrography}

Fig. 3 shows temperature and salinity against depth and temperature against salinity at each of the 2 sample sites. There are marked similarities between the profiles; at both stations a 200 to $300 \mathrm{~m}$ layer of cool, fresh ASW overlies warmer, more saline CDW and there is a pronounced temperature-minimum in the upper waters due to the seasonal warming at the surface. Fig. 1, which shows the general position of the PF in the Scotia Sea, also shows the exact position of the PF relative to Stn 1 (determined from an XBT transect between the Falkland Islands and Stn 1). Both stations were to the south of the PF.

At Stn 1 temperature decreases from $2.9^{\circ} \mathrm{C}$ at the surface to a minimum of $-0.7^{\circ} \mathrm{C}$ at $160 \mathrm{~m}$ and then increases to $2^{\circ} \mathrm{C}$ at the core of the CDW around $600 \mathrm{~m}$ (Fig. 3). There apears to be a relatively constant salinity gradient between about 100 and $400 \mathrm{~m}$. A marked change in both the temperature and salinity gradients occurs at $400 \mathrm{~m}$. Below $600 \mathrm{~m}$ temperature and salinity are relatively uniform and vary little with depth. Similar features are apparent at Stn 2 with a pronounced temperature inversion in the surface waters and salinity increasing rapidly to about $400 \mathrm{~m}$. Again, the temperature and salinity gradients alter around $400 \mathrm{~m}$ and little change in either variable is evident below $600 \mathrm{~m}$.

The water column may be subdivided as follows: (1) $0-200 \mathrm{~m}$, the bulk of the ASW with a pronounced temperature inversion; (2) $0-400 \mathrm{~m}$, the region of greatest salinity (and thus density) change; (3) 200-400 m, the depth range over which most mixing between ASW and CDW occurs; (4) $400 \mathrm{~m}$, the depth at which a marked change in temperature and salinity gradient occurs; (5) 400-600 m, the core of the CDW and the depth range over which salinity and temperature gradients are lower; (6) 600-1000 m, relatively uniform conditions.

\section{Species composition}

A total of 81 nekton and micronekton species were collected from the 2 sites. The sample size and depth range of each species, divided into day and night samples, are summarised in Table 1. Eight species, the pteropods Clione limacina, Limacina helicina, Peraclis valdiviae, Spongiobranchaea australis and Thliptodon antarcticus and the crustaceans Euphausia frigida, Thysanoessa macrura and Vibilia antarctica, were considered too small to be quantitatively sampled by the RMT 25. They are included in Table 1 but are not considered in later community analyses.

Diversity was slightly higher at Stn 2 (South Georgia slope) with 76 species, of which $19(25 \%)$ were only found there. At Stn 1 (the oceanic Scotia Sea) 62 species were recorded, of which $5(8 \%)$ were collected in that area only. Crustaceans were the most diverse group with a total of 28 species, followed by 
Station 1

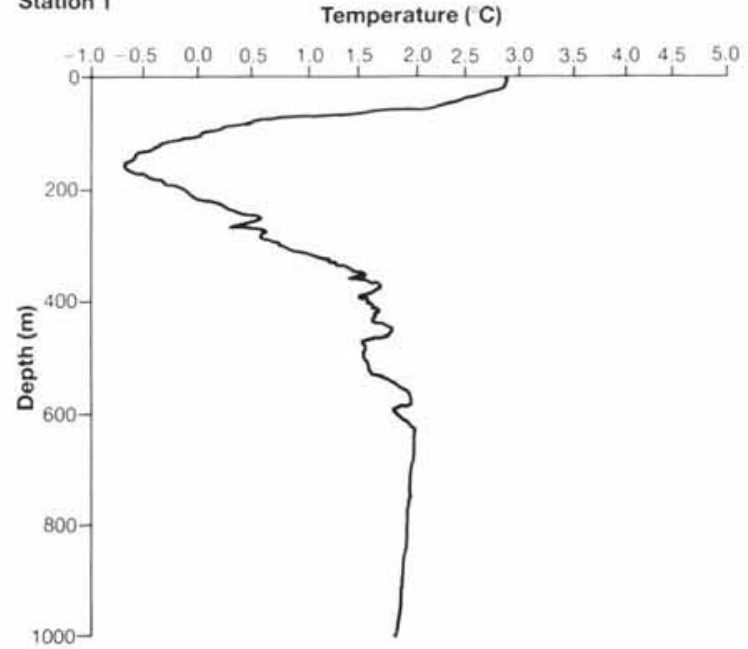

Station 1

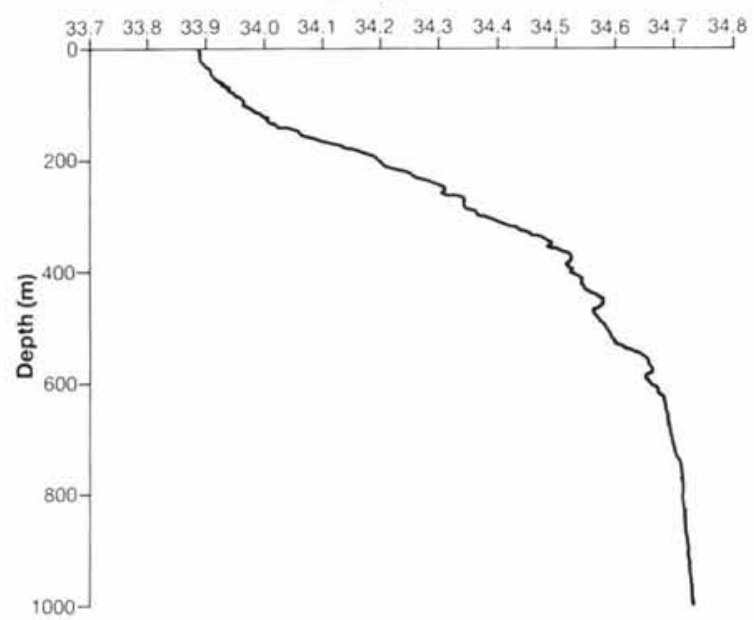

Station 1

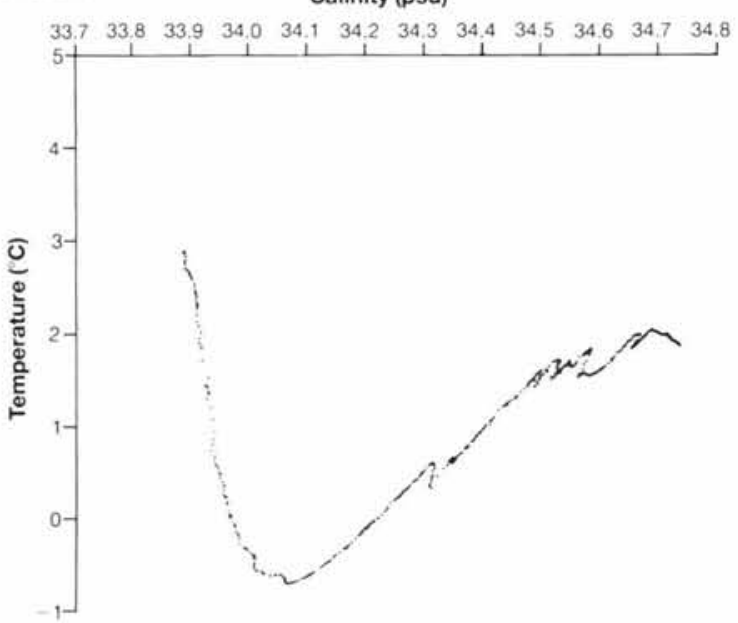

Station 2

Temperature (C)

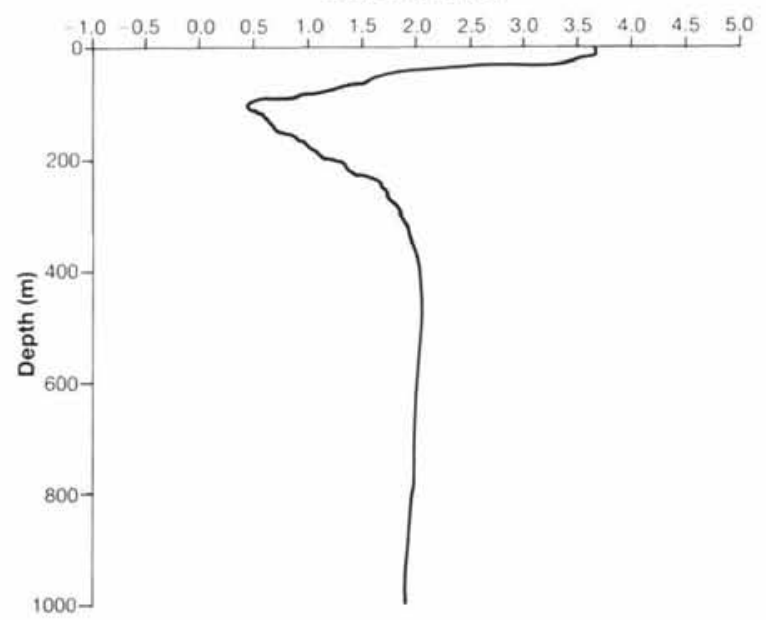

Station 2

Salinity (psu)

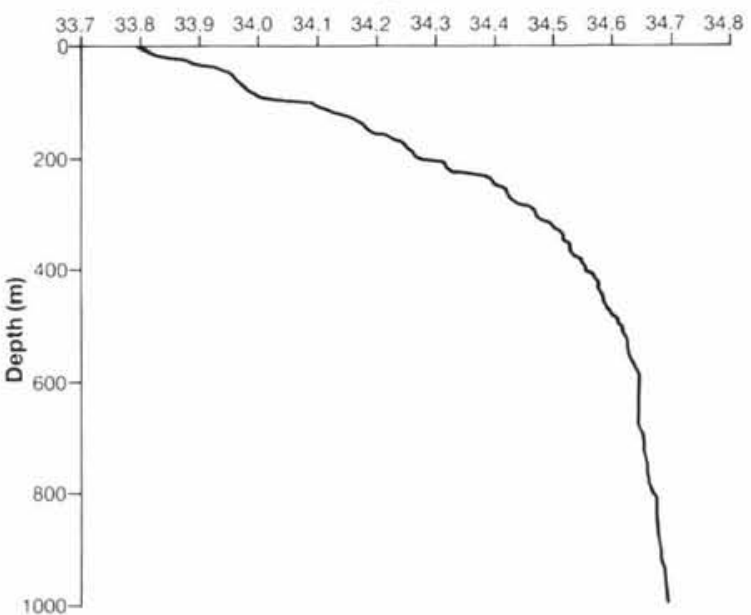

Station 2

Salinity (psu)

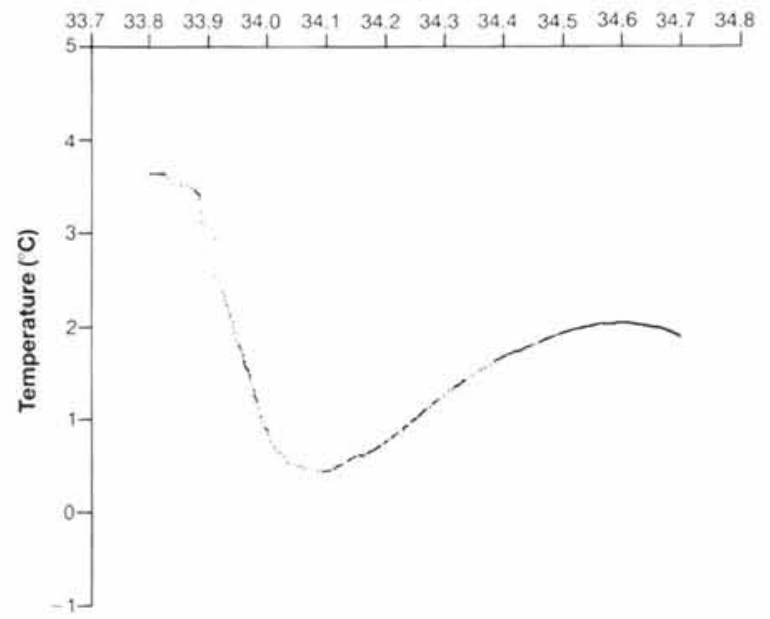

Fig. 3. Hydrographic profiles and temperature/salinity diagrams at the sample sites 
Table 1. Nekton and micronekton species list and vertical distributions. n: sample size from discrete tows. "Species not quantitatively sampled by RMT 25

\begin{tabular}{|c|c|c|c|c|c|c|c|c|}
\hline \multirow[t]{3}{*}{ Species } & \multicolumn{4}{|c|}{ Scotia Sea (Stn 1) } & \multicolumn{4}{|c|}{ South Georgia (Stn 2) } \\
\hline & \multicolumn{2}{|r|}{ Day } & \multicolumn{2}{|c|}{ Night } & \multicolumn{2}{|c|}{ Day } & \multicolumn{2}{|c|}{ Night } \\
\hline & $\mathrm{n}$ & Range (m) & $\mathrm{n}$ & Range (m) & $\mathrm{n}$ & Range (m) & $\mathrm{n}$ & Range (m) \\
\hline \multicolumn{9}{|l|}{ Coelenterata } \\
\hline Calycopsis borchgrevinki & 90 & $200-800$ & 345 & $0-800$ & 162 & $0-1000$ & 178 & $0-1000$ \\
\hline Colobonema sericeum & 1 & $800-1000$ & & - & 1 & $400-600$ & 2 & $400-800$ \\
\hline Crossota brunnea & 5 & $600-800$ & 6 & $400-600$ & 35 & $400-1000$ & 36 & $400-1000$ \\
\hline Halicreas minimum & 126 & $600-1000$ & & - & 24 & $400-1000$ & 80 & $400-1000$ \\
\hline Pantachogon haeckeli & & - & & - & & - & 22 & $200-800$ \\
\hline Hydromedusae indet. & 1 & $0-200$ & 39 & $0-200$ & 1 & $0-200$ & & - \\
\hline Atolla wyvillei & 256 & $200-1000$ & 281 & $200-1000$ & 201 & $0-1000$ & 149 & $200-1000$ \\
\hline Desmonema gaudichaudi & 2 & $200-600$ & 2 & $200-400$ & 1 & $0-200$ & & - \\
\hline Pandea rubra & 4 & $800-1000$ & & - & & - & 3 & $800-1000$ \\
\hline Periphylla periphylla & 147 & $200-1000$ & 148 & $400-1000$ & 48 & $200-1000$ & 69 & $0-1000$ \\
\hline Stygiomedusa gigantea & 1 & $200-400$ & 1 & $200-400$ & 1 & $200-400$ & 3 & $0-1000$ \\
\hline \multicolumn{9}{|l|}{ Nemertinea } \\
\hline Obnemertes maximovi & 2 & $800-1000$ & & - & 1 & $800-1000$ & 7 & $400-800$ \\
\hline \multicolumn{9}{|l|}{ Mollusca } \\
\hline Clio pyramidata & & - & & - & 3 & $200-400$ & 3 & $0-200$ \\
\hline Clio piatkowskii & 12 & $600-1000$ & 8 & $600-1000$ & 5 & $200-1000$ & 16 & $0-1000$ \\
\hline - Clione limacina & & - & 23 & $0-200$ & & - & 6 & $200-800$ \\
\hline - Limacina helicina & & - & & - & 1 & $400-600$ & 14 & $600-1000$ \\
\hline - Peraclis valdiviae & & - & & - & 1 & $400-600$ & & - \\
\hline - Spongiobranchaea australis & & - & & - & 3 & $400-1000$ & 7 & $200-600$ \\
\hline -Thliptodon antarcticus & & - & & - & & - & 1 & $400-600$ \\
\hline Alluroteuthis antarcticus & 2 & $800-1000$ & 3 & $800-1000$ & 1 & $800-1000$ & & - \\
\hline Bathyteuthis abyssicola & 1 & $800-1000$ & & - & & - & & - \\
\hline Brachioteuthis ?picta & 7 & $600-1000$ & 8 & $0-800$ & 15 & $200-1000$ & 24 & $0-1000$ \\
\hline Chiroteuthis sp. & & - & & - & 1 & $800-1000$ & & - \\
\hline Galiteuthis glacialis & 5 & $600-1000$ & 9 & $400-1000$ & 6 & $200-1000$ & 3 & $600-1000$ \\
\hline Histioteuthis eltaninae & & - & 1 & $400-600$ & & - & & - \\
\hline Mastigoteuthis psychrophila & & - & & - & 4 & $400-1000$ & 2 & $800-1000$ \\
\hline Psychroteuthis glacialis & 1 & $600-800$ & 1 & $0-200$ & & - & & - \\
\hline \multicolumn{9}{|l|}{ Polychaeta } \\
\hline Tomopteris carpenteri & & - & & - & 14 & $200-400$ & 13 & $0-400$ \\
\hline \multicolumn{9}{|l|}{ Ostracoda } \\
\hline Gigantocypris mulleri & 141 & $600-1000$ & 66 & $400-800$ & 119 & $200-1000$ & 78 & $200-1000$ \\
\hline \multicolumn{9}{|l|}{ Euphausiacea } \\
\hline •Euphausia frigida & 3 & $400-600$ & & - & 104 & $0-200$ & 98 & $0-200$ \\
\hline Euphausia superba & 13 & $400-600$ & 4 & $200-600$ & 112 & $0-800$ & 95 & $0-800$ \\
\hline Euphausia triacantha & 1944 & $0-1000$ & 453 & $0-800$ & 2333 & $0-1000$ & 10833 & $0-800$ \\
\hline - Thysanoessa macrura & & - & 56 & $0-600$ & 313 & $0-200$ & 127 & $0-400$ \\
\hline \multicolumn{9}{|l|}{ Decapoda } \\
\hline Acanthephyra pelagica & 26 & $200-400$ & 17 & $400-600$ & 42 & $200-800$ & 41 & $200-1000$ \\
\hline Gennadas kempi & 15 & $600-1000$ & & - & 20 & $400-1000$ & 39 & $400-800$ \\
\hline Pasiphaea grandicula & 1 & $600-800$ & 21 & $0-800$ & 2 & $400-1000$ & 3 & $400-800$ \\
\hline Pasiphaea scotiae & 42 & $600-1000$ & 86 & $200-1000$ & 48 & $200-1000$ & 35 & $200-1000$ \\
\hline Petalidium foliaceum & 66 & $600-1000$ & 8 & $800-1000$ & 54 & $400-1000$ & 32 & $200-1000$ \\
\hline Sergestes arcticus & 11 & $600-800$ & & - & 1 & $400-600$ & 2 & $400-600$ \\
\hline Stereomastis suhmi & & - & 1 & $600-800$ & 4 & $400-800$ & & - \\
\hline \multicolumn{9}{|l|}{ Mysidacea } \\
\hline Boreomysis sp. & & - & & - & 1 & $800-1000$ & 1 & $600-800$ \\
\hline Gnathophausia gigas & 21 & $600-1000$ & 19 & $200-1000$ & 12 & $400-1000$ & 14 & $400-1000$ \\
\hline
\end{tabular}


Table 1 (continued)

\begin{tabular}{|c|c|c|c|c|c|c|c|c|}
\hline \multirow[t]{3}{*}{ Species } & \multicolumn{4}{|c|}{ Scotia Sea (Stn 1) } & \multicolumn{4}{|c|}{ South Georgia (Stn 2) } \\
\hline & \multicolumn{2}{|c|}{ Day } & \multicolumn{2}{|c|}{ Night } & \multicolumn{2}{|c|}{ Day } & \multicolumn{2}{|c|}{ Night } \\
\hline & $\mathrm{n}$ & Range (m) & $\mathrm{n}$ & Range (m) & $\mathrm{n}$ & Range (m) & $\mathrm{n}$ & Range (m) \\
\hline \multicolumn{9}{|l|}{ Amphipoda } \\
\hline Cyphocaris richardi & 44 & $600-1000$ & 173 & $0-1000$ & 117 & $400-1000$ & 129 & $200-1000$ \\
\hline Danaella mimonectes & & - & & - & & - & 2 & $200-600$ \\
\hline Eurythenes obesus & & - & & - & 11 & $400-1000$ & 9 & $0-1000$ \\
\hline Eusiroides stenopleura & 7 & $800-1000$ & 3 & $400-600$ & 3 & $400-1000$ & 7 & $400-800$ \\
\hline Parandania boecki & 43 & $600-1000$ & 26 & $200-800$ & 72 & $400-1000$ & 110 & $200-1000$ \\
\hline Cyllopus lucasii & 21 & $0-600$ & 15 & $200-600$ & 92 & $0-600$ & 75 & $0-600$ \\
\hline Hyperia antarctica & 8 & $400-600$ & 4 & $600-800$ & 6 & $400-800$ & 3 & $200-800$ \\
\hline Hyperia macrocephala & & - & & - & & - & 3 & $0-200$ \\
\hline Lanceola sp. & 2 & $200-1000$ & & - & 1 & $800-1000$ & 3 & $600-1000$ \\
\hline Megalolanceola stephenseni & 1 & $800-1000$ & & - & & - & & - \\
\hline Pegohyperia princeps & 5 & $600-800$ & 4 & $600-1000$ & 5 & $400-1000$ & 8 & $200-1000$ \\
\hline Scypholanceola aestiva & & - & & - & & - & 1 & $400-600$ \\
\hline Themisto gaudichaudii & 108 & $0-800$ & 266 & $0-1000$ & 13287 & $0-1000$ & 6570 & $0-800$ \\
\hline -Vibilia antarctica & & - & & - & 2 & $200-400$ & 7 & $0-600$ \\
\hline \multicolumn{9}{|l|}{ Tunicata } \\
\hline Salpa thompsoni & 5361 & $0-1000$ & 17297 & $0-1000$ & 8 & $0-800$ & 7 & $0-800$ \\
\hline \multicolumn{9}{|l|}{ Vertebrata (Pisces) } \\
\hline Nansenia antarctica & 15 & $400-800$ & 6 & $400-800$ & 6 & $0-800$ & 9 & $200-600$ \\
\hline Bathylagus antarcticus & 43 & $600-1000$ & 275 & $200-1000$ & 70 & $200-1000$ & 42 & $0-1000$ \\
\hline Cyclothone spp. & 48 & $400-1000$ & 16 & $0-1000$ & 17 & $400-1000$ & 48 & $200-1000$ \\
\hline Borostomias antarcticus & 4 & $600-800$ & 6 & $0-1000$ & 9 & $200-800$ & 6 & $200-1000$ \\
\hline Stomias ? gracilis & 1 & $600-800$ & 8 & $0-600$ & & - & 1 & $400-600$ \\
\hline Benthalbella elongata & 5 & $600-1000$ & 1 & $600-800$ & 2 & $400-800$ & 9 & $200-1000$ \\
\hline Notolepis coatsi & 5 & $400-1000$ & 8 & $400-800$ & 7 & $200-800$ & 13 & $0-1000$ \\
\hline Electrona antarctica & 67 & $200-1000$ & 125 & $0-1000$ & 202 & $0-1000$ & 161 & $0-1000$ \\
\hline Electrona carlsbergi & 7 & $600-800$ & 89 & $0-800$ & 5 & $0-600$ & 4 & $200-600$ \\
\hline Gymnoscopelus bolini & & - & 4 & $600-1000$ & 2 & $200-600$ & 3 & $200-800$ \\
\hline Gymnoscopelus braueri & 74 & $400-1000$ & 162 & $0-1000$ & 99 & $400-1000$ & 274 & $0-800$ \\
\hline Gymnoscopelus fraseri & & - & 7 & $0-200$ & 14 & $0-1000$ & 1 & $0-200$ \\
\hline Gymnoscopelus nicholsi & 1 & $800-1000$ & & - & 12 & $0-600$ & 7 & $0-200$ \\
\hline Krefftichthys anderssoni & 53 & $0-1000$ & 229 & $200-1000$ & 277 & $0-1000$ & 200 & $0-1000$ \\
\hline Lampanyctus achirus & 10 & $600-800$ & 12 & $400-1000$ & 14 & $400-1000$ & 19 & $200-1000$ \\
\hline Protomyctophum andriashevi & & - & & - & & - & 1 & $0-200$ \\
\hline Protomyctophum bolini & 75 & $200-800$ & 131 & $0-800$ & 132 & $0-800$ & 130 & $0-600$ \\
\hline Protomyctophum choriodon & & - & & - & 8 & $0-600$ & 36 & $0-200$ \\
\hline Protomyctophum gemmatum & $n \quad 5$ & $400-600$ & 2 & $400-600$ & & - & & - \\
\hline Melanonus gracilis & & - & & - & 1 & $400-600$ & & - \\
\hline Nemichthys ?scolopaceus & 1 & $400-600$ & & - & 2 & $400-800$ & & - \\
\hline Cyanomacrurus piriei & 2 & $800-1000$ & 2 & $800-1000$ & 5 & $400-1000$ & 9 & $400-1000$ \\
\hline Poromitra crassiceps & 9 & $600-1000$ & 4 & $800-1000$ & 9 & $200-1000$ & 8 & $600-1000$ \\
\hline Paradiplospinus gracilis & & - & & - & 4 & $200-1000$ & 4 & $0-1000$ \\
\hline
\end{tabular}

mesopelagic fish with 24 species. Molluscs were represented by 15 species, of which 8 were cephalopods. Coelenterates occurred with 11 species. Two of them, Desmonema gaudichaudi and Stygiomedusa gigantea, were large scyphomedusae.

The tunicate Salpa thompsoni was present in very dense aggregations at Stn 1 and outnumbered all other species (Table 1). It was relatively scarce at Stn 2. At Stn 1 the crustaceans were dominated by the euphausiid Euphausia triacantha, whereas the hyperiid amphipod Themisto gaudichaudii was the most abundant crustacean at Stn 2. No dense swarms of the Antarctic krill Euphausia superba were present in the areas investigated. Fish numbers were dominated by myctophids (Electrona antarctica, Gymnoscopelus braueri, Krefftichthys anderssoni) at both stations.

\section{Vertical distribution of numerically dominant species}

Most species occurred over a wide depth range and were caught at depths down to $1000 \mathrm{~m}$, with numbers 

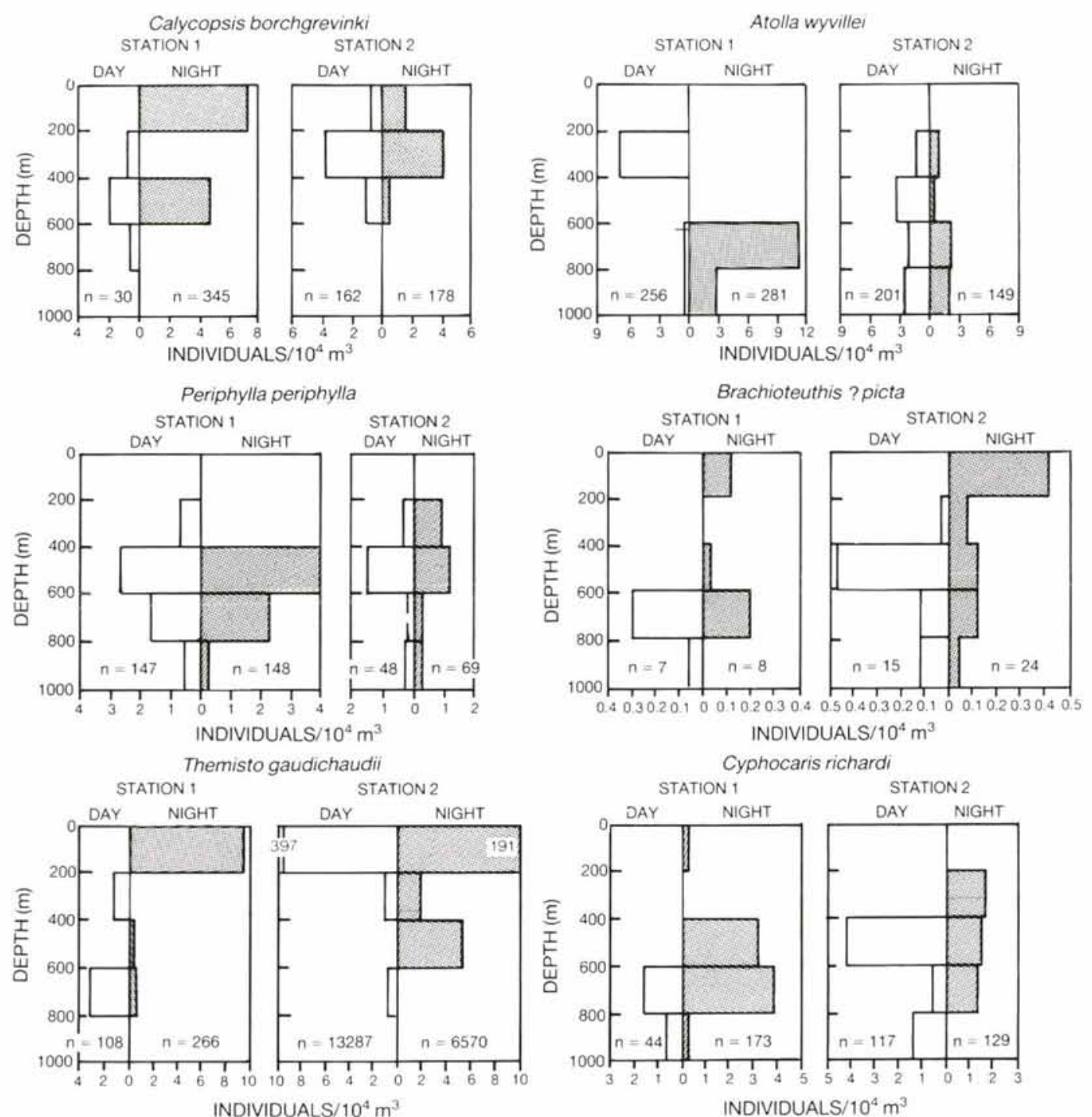

Fig. 4 (this and facing page). Vertical distribution of numerically most abundant species. n: no. of specimens collected
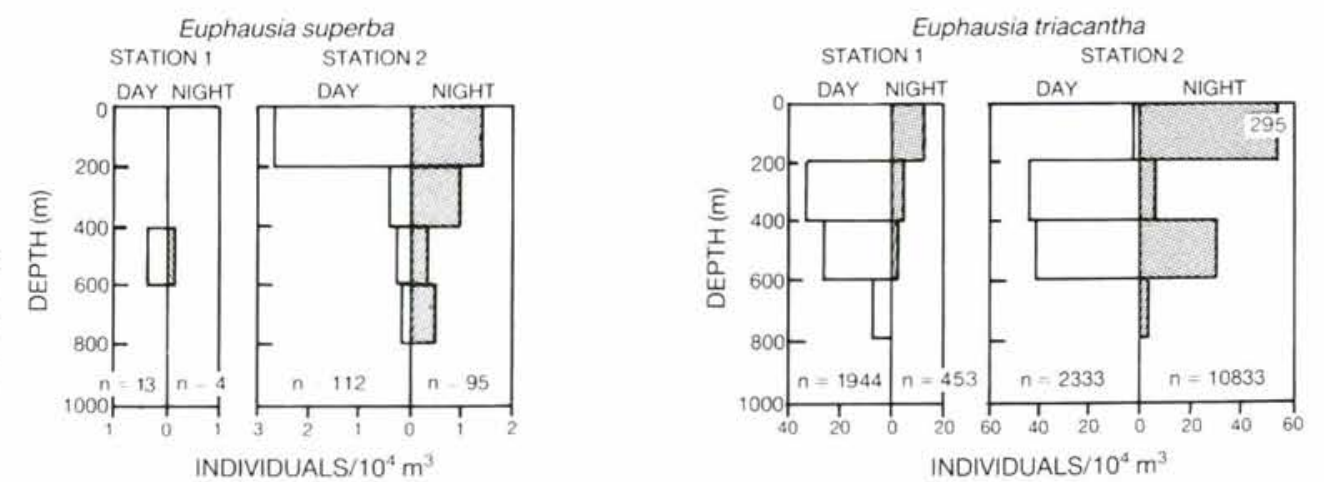

decreasing in the deepest layer during night hauls. The majority of species were not present in the upper $200 \mathrm{~m}$ (Table 1), although the number of species caught in this layer increased during the night. Most species were rare, i.e. $<1$ ind. $10^{-4} \mathrm{~m}^{-3}$.

The vertical depth ranges of 16 major nekton species (Fig. 4) suggest some distinct diel vertical migratory patterns. With the exception of Atolla wyvillei, Periphylla periphylla, Cyphocaris richardi, Gigantocypris mulleri and Pasiphaea scotiae which had their abun- 

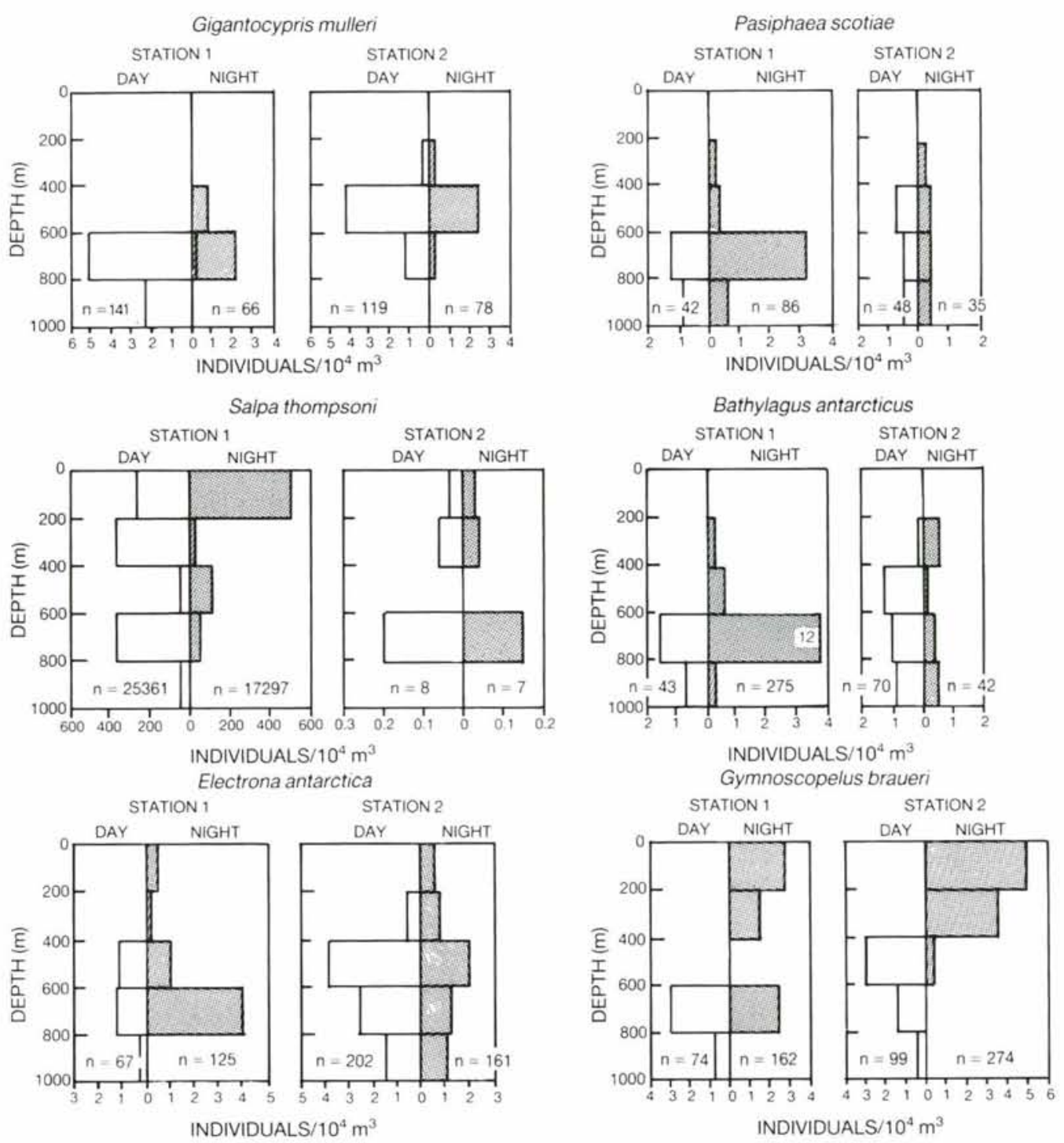

Krefftichthys anderssoni
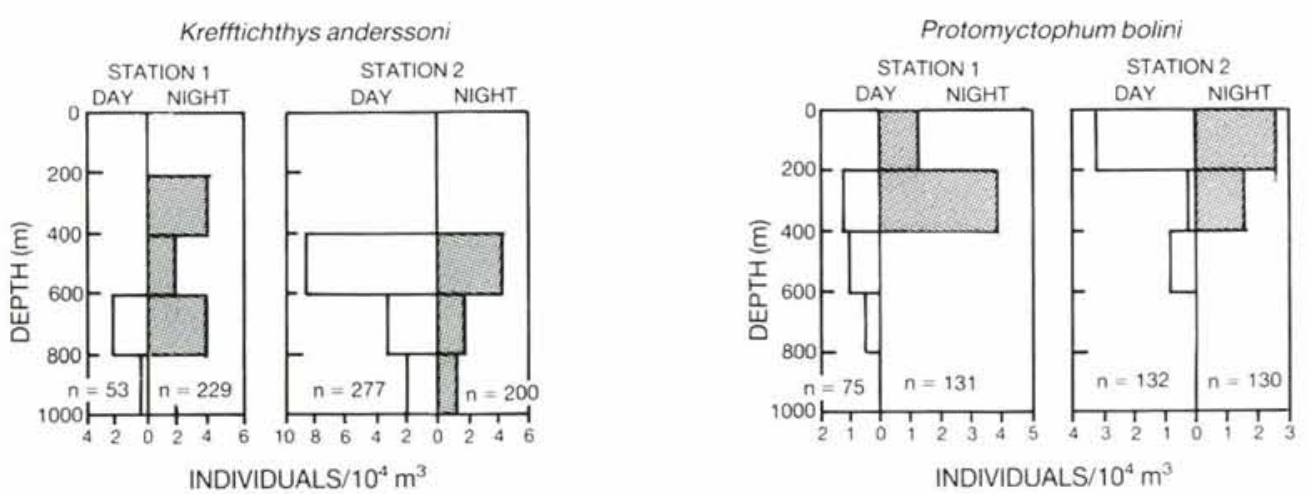

dance peaks in the CDW and showed no clear indication of a vertical movement, all species exhibited an upward migration during the night.

The hydromedusa Calycopsis borchgrevinki showed a strong vertical migration at Stn 1 where it ascended from the 200-600 m layers during the day to the upper 0-200 m layer during night. The squid Brachioteuthis ?picta occurred mainly below $600 \mathrm{~m}$ at Stn 1; at Stn 2 it showed a pronounced vertical migration from the CDW during the day into the surface ASW during the 
night. Euphausia superba, very rare at Stn 1, was quite common at Stn 2 with higher concentrations in the surface layer during daylight than at night. At both stations $E$. triacantha showed a pronounced vertical migration into the surface layer during the night, where it was nearly absent during daylight. The very abundant tunicate Salpa thompsoni exhibited a strong vertical migration at Stn 1; during daylight it was distributed over a wide depth range while at night it concentrated in the upper $0-200 \mathrm{~m}$.

All the common mesopelagic fish migrated vertically. At both stations the myctophids Electrona antarctica, Gymnoscopelus braueri, Krefftichthys anderssoni, Protomyctophum bolini and the bathylagid Bathylagus antarcticus ascended from the deeper layers during daylight to the surface layers during night. This pattern was particularly marked for $G$. braueri, but only weakly exhibited by $B$. antarcticus.
Table 2. Nekton biomass from RMT 25 samples. Standing stock in $\mathrm{g}$ wet $\mathrm{wt} \mathrm{m}^{-2}$

\begin{tabular}{|crrrr|}
\hline \multirow{2}{*}{$\begin{array}{c}\text { Depth range } \\
(\mathrm{m})\end{array}$} & \multicolumn{2}{c}{ Stn 1 } & \multicolumn{2}{c|}{ Stn 2 } \\
& Day & Night & Day & Night \\
\hline $0-200$ & 25.2 & 33.0 & 1.2 & 3.6 \\
$200-400$ & 37.2 & 17.8 & 1.2 & 2.6 \\
$400-600$ & 4.4 & 12.0 & 1.2 & 5.0 \\
$600-800$ & 21.8 & 19.2 & 3.0 & 4.7 \\
$800-1000$ & 6.0 & 5.0 & 3.6 & 7.8 \\
$0-1000$ & 94.6 & 87.0 & 10.2 & 23.7 \\
\hline
\end{tabular}

absent. At both sites coelenterates (Periphylla periphylla, Atolla wyvillei) and mesopelagic fish (Bathylagus antarcticus, Electrona antarctica, Gymnoscopelus braueri) comprised the majority of the biomass. The crustaceans Euphausia triacantha and Themisto gaudichaudii were major contributors at Stn 2 only (Table 3 ).

\section{Standing stock biomass}

The total nekton biomass per unit area ( $\mathrm{g}$ wet wt $\mathrm{m}^{-2}$ ) for each $200 \mathrm{~m}$ layer is given in Table 2. At Stn 1 the maximum nekton biomass occurred during daylight in the 2 upper layers, with evidence of diel vertical migration from $200-400 \mathrm{~m}$ during daylight $\left(37.2 \mathrm{~g}\right.$ wet $\left.\mathrm{wt} \mathrm{m}^{-2}\right)$ to $0-200 \mathrm{~m}$ at night $\left(33.0 \mathrm{~g}\right.$ wet $\left.\mathrm{wt} \mathrm{m}^{-2}\right)$. There was also a pronounced peak in biomass in the 600-800 m layer in both daylight and darkness (21.8 and $19.2 \mathrm{~g}$ wet wt $\mathrm{m}^{-2}$, respectively), although there was no evidence of diel vertical migration at this depth. At Stn 2, the nekton biomass was much lower with a maximum of $7.8 \mathrm{~g}$ wet wt $\mathrm{m}^{-2}$ at $800-1000 \mathrm{~m}$ during the night. There was little evidence of diel vertical migration between any of the layers but biomass in all layers was consistently higher in hauls made during the night than in daylight, which suggests an upward migration into the upper $1000 \mathrm{~m}$ from layers below.

The standing stock values (mg wet wt $\mathrm{m}^{-2}$ ) for the major taxonomic groups and the most abundant species illustrate that only a relatively few species constitute the majority of the biomass (Table 3 ). At Stn 1 the tunicate Salpa thompsoni was the major species in terms of biomass (41570 mg wet wt $\mathrm{m}^{-2} ; 0-1000 \mathrm{~m}$ ); however, at $\operatorname{Stn} 2$ the species was virtually
Table 3. Biomass (mg wet wt $\mathrm{m}^{-2}$ ) of major taxonomic groups and selected dominant species calculated from RMT 25 hauls performed during night

\begin{tabular}{|c|c|c|c|c|}
\hline \multirow[t]{2}{*}{ Group/Species } & \multicolumn{2}{|c|}{ Stn 1} & \multicolumn{2}{|c|}{$\operatorname{Stn} 2$} \\
\hline & $0-200 \mathrm{~m}$ & $0-1000 \mathrm{~m}$ & $0-200 \mathrm{~m}$ & $0-1000 \mathrm{~m}$ \\
\hline Coelenterata & 462 & 28333 & 725 & 15126 \\
\hline Mollusca & 18 & 235 & 28 & 158 \\
\hline Euphausiacea & 24 & 41 & 1197 & 1461 \\
\hline Decapoda & 41 & 633 & 0 & 336 \\
\hline Amphipoda & 2 & 137 & 769 & 895 \\
\hline Tunicata $^{a}$ & 28632 & 41570 & 2 & 11 \\
\hline Pisces & 487 & 4922 & 489 & 4216 \\
\hline Others $^{b}$ & 3334 & 11129 & 390 & 1497 \\
\hline Calycopsis borchgrevinki & 150 & 423 & 54 & 277 \\
\hline Atolla wyvillei & 0 & 9694 & 0 & 1641 \\
\hline Periphylla periphylla & 0 & 10675 & 15 & 11117 \\
\hline Brachioteuthis ?picta & 17 & 63 & 25 & 75 \\
\hline Gigantocypris mulleri & 0 & 73 & 0 & 64 \\
\hline Euphausia triacantha & 24 & 38 & 1180 & 1420 \\
\hline Acanthephyra pelagica & 0 & 79 & 0 & 223 \\
\hline Pasiphaea scotiae & 0 & 510 & 0 & 46 \\
\hline Cyphocaris richardi & 2 & 72 & 0 & 26 \\
\hline Parandania boecki & 0 & 59 & 0 & 35 \\
\hline Themisto gaudichaudii & 28 & 35 & 766 & 781 \\
\hline Bathylagus antarcticus & 0 & 2361 & 9 & 628 \\
\hline Borostomias antarcticus & 4 & 103 & 0 & 176 \\
\hline Electrona antarctica & 39 & 441 & 57 & 895 \\
\hline Electrona carlsbergi & 270 & 292 & 0 & 27 \\
\hline Gymnoscopelus bolini & 0 & 219 & 0 & 228 \\
\hline Gymnoscopelus braueri & 62 & 302 & 243 & 817 \\
\hline Krefftichthys anderssoni & 0 & 486 & 1 & 309 \\
\hline Protomyctophum bolini & 54 & 164 & 74 & 101 \\
\hline Cyanomacrurus piriei & 0 & 175 & 0 & 309 \\
\hline
\end{tabular}




\section{Community analysis}

The results of the cluster analysis are shown in a dendrogram (Fig. 5). The ordinate represents the groupings or clusters of samples and the abscissa the percentage similarity. The samples are grouped according to both presence and abundance of species. Thus, the clusters derive from the similarity of the nekton composition in the different samples and reflect the different nekton communities responsible for the sample groupings.

At an arbitrary similarity level of $40 \%, 4$ major net haul clusters and 1 single sample emerge (Fig. 5). Four communities can be derived. Cluster 1 groups the 4 deepest nekton samples $(800-1000 \mathrm{~m})$ of both stations plus the night sample for the 600-800 m layer at Stn 2 . It represents the lower mesopelagic nekton community. Cluster 2 represents samples from the $400-600 \mathrm{~m}$ and 600-800 $\mathrm{m}$ layers of both Stns 1 and 2. It represents an upper nekton community, and although similar to the lower mesopelagic community, it is richer in species and biomass. In Cluster 3 the 4 net hauls from

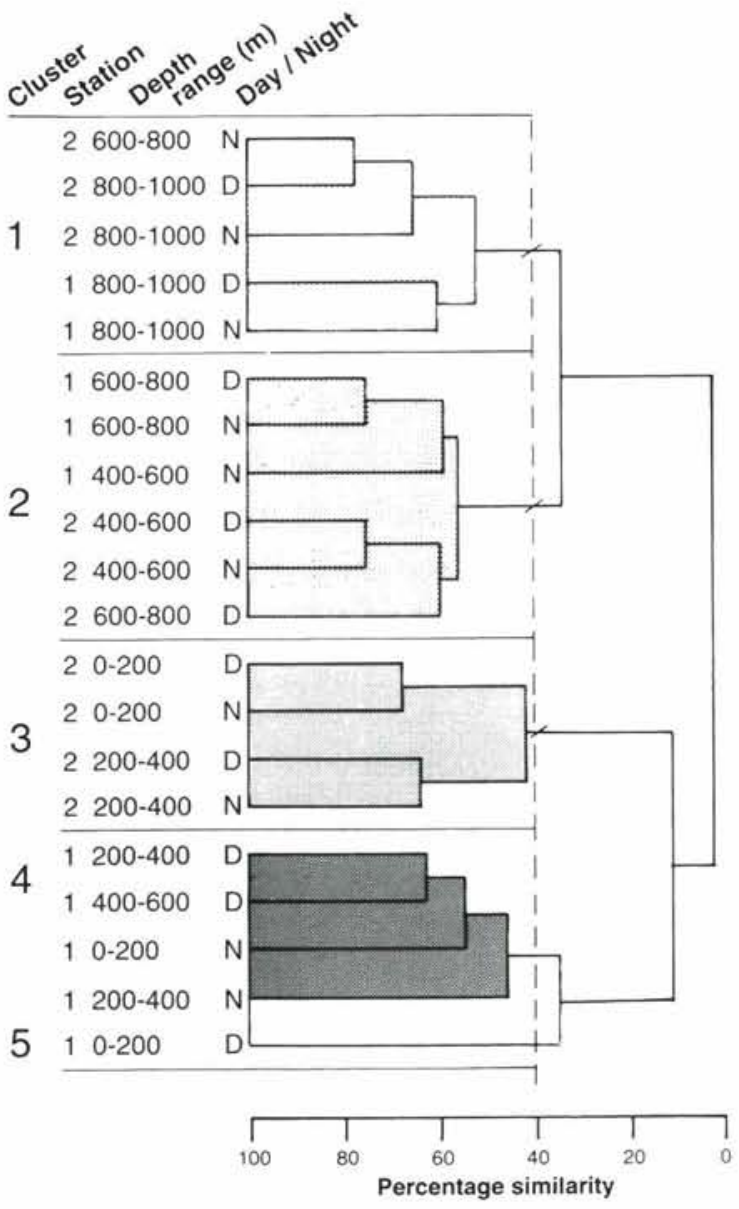

Fig. 5. Cluster analysis dendrogram (Bray-Curtis Index, complete linkage)

\begin{tabular}{|c|c|c|c|c|}
\hline \multirow{3}{*}{$\frac{m}{0-200}$} & \multicolumn{2}{|c|}{ Station 1} & \multicolumn{2}{|c|}{ Station 2} \\
\hline & DAY & NIGHT & DAY & NIGHT \\
\hline & 5 & 4 & 3 & 3 \\
\hline $200-400$ & 4 & 4 & 3. & 3 \\
\hline $400-600$ & 4 & 2 & 2 & 2 \\
\hline $600-800$ & 2. & 2 & 2 & 11 \\
\hline $800-1000$ & 1. & 1 & 11 & 11 \\
\hline
\end{tabular}

Fig. 6. Extension of the nekton communities as revealed by cluster analysis (see Fig. 5)

the upper layers $(0-200 \mathrm{~m}, 200-400 \mathrm{~m})$ of Stn 2 are grouped together. This cluster characterises the epipelagic $(0-400 \mathrm{~m})$ nekton community of the South Georgia slope region. Cluster 4 is a similar grouping, but is restricted to samples from Stn 1. This cluster also includes the daylight sample from the 400-600 m layer at Stn 1 and describes the epipelagic nekton community of the oceanic Scotia Sea. The daylight sample from the upper surface layer $(0-200 \mathrm{~m})$ of Stn 1 was an outlier which could not be grouped into any of the existing clusters. A diagram of the distribution of communities is given in Fig. 6 .

The characteristics of the assemblages in each depth/time stratum in terms of the number of species, the dominant species and its percentage dominance and a diversity index (Shannon \& Weaver 1963) were compiled for each sample (Table 4). At Stn 1 Salpa thompsoni was the dominant species in all samples. This species was of low importance at Stn 2, where the dominant species varied for the different layers. Species number and diversity increased with depth and reached higher values at Stn 2 .

\section{DISCUSSION}

\section{Net performance}

In calculating nekton relative abundance and biomass densities from RMT 25 samples, it should be noted that the effective mouth area of the net is dependent upon the mouth angle during fishing. The nominal mouth area of $25 \mathrm{~m}^{2}$ is only achieved when the mouth angle is $45^{\circ}$. The actual mouth area is dependent on towing speed since the bottom bar weight remains constant but the net drag is proportional to the square of velocity. Roe et al. (1980) showed that for an RMT 8 there is a speed range over which the mouth angle remains constant (2.6 to 8.0 knots). Our net, having larger meshes making up the majority of its filtering area, would be expected to have a proportionally lower drag and so reach a similar mouth angle plateau at a higher speed. Thus, for horizontal towing at 
Table 4. Number of species, dominant species, its percentage dominance and diversity index $H^{\prime}=-\Sigma(n / N) \ln (n / N)$ after Shannon (Shannon \& Weaver 1963) with $n=$ relative abundance of each species in the sample and $N=$ total relative abundance of all species in the sample

\begin{tabular}{|c|c|c|c|c|c|c|}
\hline Stn & $\begin{array}{l}\text { Sampling } \\
\text { depth (m) }\end{array}$ & $\begin{array}{c}\text { Day or } \\
\text { night }\end{array}$ & $\begin{array}{l}\text { No. of } \\
\text { species }\end{array}$ & Dominant species & $\begin{array}{l}\text { Percentage } \\
\text { dominance }\end{array}$ & $\begin{array}{c}\text { Diversity } \\
\text { index }\end{array}$ \\
\hline 1 & $0-200$ & D & 6 & Salpa thompsoni & 99.8 & 0.017 \\
\hline 1 & $0-200$ & $\mathrm{~N}$ & 17 & Salpa thompsoni & 92.8 & 0.391 \\
\hline 1 & $200-400$ & D & 13 & Salpa thompsoni & 88.8 & 0.460 \\
\hline 1 & $200-400$ & $\mathrm{~N}$ & 21 & Salpa thompsoni & 54.4 & 1.611 \\
\hline 1 & $400-600$ & $\mathrm{D}$ & 17 & Salpa thompsoni & 53.8 & 1.151 \\
\hline 1 & $400-600$ & $N$ & 28 & Salpa thompsoni & 84.7 & 0.803 \\
\hline 1 & $600-800$ & D & 36 & Salpa thompsoni & 89.6 & 0.635 \\
\hline 1 & $600-800$ & $N$ & 31 & Salpa thompsoni & 49.1 & 1.912 \\
\hline 1 & $800-1000$ & $\mathrm{D}$ & 34 & Salpa thompsoni & 66.2 & 1.531 \\
\hline 1 & $800-1000$ & $\mathrm{~N}$ & 22 & Salpa thompsoni & 41.5 & 2.088 \\
\hline 2 & $0-200$ & $\mathrm{D}$ & 16 & Themisto gaudichaudii & 97.4 & 0.171 \\
\hline 2 & $0-200$ & $N$ & 25 & Euphausia triacantha & 58.7 & 0.859 \\
\hline 2 & $200-400$ & $\mathrm{D}$ & 27 & Euphausia triacantha & 80.3 & 0.946 \\
\hline 2 & $200-400$ & $N$ & 35 & Euphausia triacantha & 22.4 & 2.613 \\
\hline 2 & $400-600$ & $\mathrm{D}$ & 48 & Euphausia triacantha & 49.2 & 2.132 \\
\hline 2 & $400-600$ & $\mathrm{~N}$ & 44 & Euphausia triacantha & 50.5 & 2.105 \\
\hline 2 & $600-800$ & $\mathrm{D}$ & 35 & Krefftichthys anderssoni & 18.7 & 2.741 \\
\hline 2 & $600-800$ & $N$ & 42 & Euphausia triacantha & 15.8 & 2.951 \\
\hline 2 & $800-1000$ & $\mathrm{D}$ & 37 & Petalidium foliaceum & 13.7 & 2.883 \\
\hline 2 & $800-1000$ & $\mathrm{~N}$ & 32 & Atolla wyvillei & 19.5 & 2.795 \\
\hline
\end{tabular}

3 knots we are confident that the mouth angle was close to $45^{\circ}$. However, exact information on the mouth angle of the net can only be achieved if the inclination of the net will be measured directly during the haul.

With the mouth at its design angle the bottom bar trails $5 \mathrm{~m}$ behind the top bar. Oblique haul techniques will thus increase mouth area during downward hauls and decrease mouth area during upward hauls.

With the larger mesh panels at the front of the net the $25 \mathrm{~m}^{2}$ mouth area may not be appropriate for calculating the densities of those species able to escape through the larger mesh sizes. However, given the probability that the net will exert a herding effect this problem is complex and cannot be addressed further in the present study.

Finally, the performance of the RMT 25 may also be influenced by the angled mouth design in that objects, such as rigging wires, monitor and release gear, pass over the organisms and, if detected, may elicit an escape response before the organisms actually enter the net. Net avoidance is most likely to be a problem in well lit water but it may also occur at depth where bioluminescence, stimulated by the net gear, may indicate the presence of the net.

\section{Distribution patterns and community structure}

The composition of pelagic ecosystems varies considerably in space and time, resulting in an extremely heterogeneous distribution of key species (Haury et al.
1978, Omori \& Hamner 1982). Thus, the results of the present study obtained from hauls at 2 considerably different sites within the Scotia Sea and during only 6 d of January 1991 should be considered simply as a snapshot in time of one important trophic level of the Antarctic pelagic ecosystem. Our study provides a sound basis for any further investigation of the Antarctic nekton community, a previously widely ignored element of the Southern Ocean ecosystem, even though restricted spatially and temporally. In fact, the opening/closing version of the RMT 25 revealed for the first time detailed information on the vertical structure and species-specific biomass levels of the Antarctic nekton community.

The present study is the first examination of the summer nekton community in the Scotia Sea and provides the most comprehensive nekton and micronekton species list reported for the Southern Ocean. It was found that large numbers of mesopelagic fish which also contained a number of rare forms (Gon \& Heemstra 1990) were responsible for the high nekton diversity. Both Stns 1 and 2 were situated outside the zone influenced regularly by seasonal pack ice, in which Euphausia superba, the Antarctic krill, dominates the pelagic ecosystem (Hempel 1987) and so, as expected, relatively small numbers of $E$. superba were recorded. However, krill distribution is notoriously patchy and we may, by chance, have missed sampling through a swarm leading to a low recorded abundance in this study. Mesopelagic fish, salps, coelenterates, decapod crustaceans and amphipods were the predominant 
nekton organisms, both in terms of numerical abundance and biomass. These typical nekton groups have been observed to form distinct faunal assemblages in midwater (e.g. Auster et al. 1992) and, if in high densities, are responsible for the development of so-called deep-scattering layers (Milliman \& Manheim 1968).

At both sites our species list described a typical Antarctic oceanic nekton fauna which included several species previously reported from adjacent regions (Mackintosh 1934, Lancraft et al. 1989, 1991). This list also contained a number of species, such as the nemertean Obnemertes maximovi, the decapods Pasiphaea grandicula and Sergestes arctica, and the hyperiid amphipods Megalolanceola stephensi and Scypholanceola aestiva which have been reported only sporadically from the Southern Ocean.

At both stations species diversity increased considerably with depth (Table 4) and this is attributed to the species-rich CDW below about $400 \mathrm{~m}$ (Fig. 3). A number of typical mesopelagic species occurred there which avoided the surface layers (i.e. the scyphomedusae Atolla wyvillei and Periphylla periphylla, the squid Mastigoteuthis psychrophila, the ostracod Gigantocypris mulleri, the decapod Gennadas kempi, the amphipod Eusiroides stenopleura, and the fish Cyanomacrurus piriei and Poromitra crassiceps).

One of the most striking results from our studies was that cluster analysis grouped samples from the CDW (400-1000 m) from both study sites into the same communities (Clusters 1 and 2; Fig. 5). In contrast, samples from the upper layers $(0-400 \mathrm{~m})$, which were characterised by ASW and waters where mixing between ASW and CDW occurred, were geographically separate (Clusters 3 and 4 ; Fig. 5).

The communities present at Stns 1 and 2 were the lower mesopelagic nekton community, with numerous species typical of the deep layer (800-1000 m) of CDW (Cluster 1), and the upper mesopelagic nekton community mainly restricted to the 400-800 m layers of CDW (Cluster 2). The latter, higher depth strata, were richer in biomass and species abundance than the lower CDW level (Table 2, Fig. 4). We attribute this to (1) most mesopelagic species having their peak distribution in the core of the CDW in about $500-600 \mathrm{~m}$, and (2) the diurnal migration of several species from the deepest layers into the 400-800 m layers. However, this pattern was not consistent. At Stn 1 the daylight 400-600 m sample was grouped in the surface cluster (Cluster 4 ) by its low species number, and at Stn 2 the nighttime $600-800 \mathrm{~m}$ sample was grouped with the lower mesopelagic community (Cluster 1) probably by the high portion of mesopelagic species that have their distribution centre in the deeper layers.

The surface layers were clearly geographically separate, although their large-scale hydrography was similar. ASW characterised the upper $200-300 \mathrm{~m}$ of the water column at both stations, with a seasonally warmed upper surface layer of ca $60 \mathrm{~m}\left(>2.0^{\circ} \mathrm{C}\right)$. Higher species numbers and diversities caused by the proximity of South Georgia (the 'island effect') probably explained the conspicuous grouping of the slope samples into the South Georgia epipelagic nekton community (Cluster 3). Small-sized species such as the pteropod Clio pyramidata and the polychaete Tomopteris carpenteri were only found in this community. Themisto gaudichaudii and Euphausia triacantha were numerically very abundant and $E$. superba, known to be typical in the area (Ward et al. 1990), was also a common component (Table 1, Fig. 4). Although close to the shelf of South Georgia this community should not be considered neritic as no benthic, benthopelagic or meroplanktonic stages of typical demersal species were present.

The surface samples of Stn 1 were grouped into the epipelagic community of the Scotia Sea (Cluster 4) which also included the daylight sample at 400-600 m layer as described above. This community presented an impoverished fauna that was less diverse than the corresponding community at South Georgia and considerably affected by large concentrations of Salpa thompsoni. Numerically this tunicate dominated all samples at Stn 1 (Table 4) while at Stn 2 it was virtually absent. $S$. thompsoni is a highly variable component in the pelagic system of the Southern Ocean (Everson 1984, Piatkowski 1985a, b) and its locally restricted, high abundance had a strong influence when characterising the Stn 1 community. The species is extremely patchy and can outnumber all other species by a smallscale rapid multiplication through asexual reproduction during times of favourable food supply, i.e. algal blooms (Foxton 1966). The relatively clear separation of the communities (Clusters 1 to 4 ) was biased by one outlier sample. The daylight $0-200 \mathrm{~m}$ catch at Stn 1 was grouped separately from all other samples due to the lowest species number and diversity encountered (Table 4).

Biomass levels were expressed in wet weight to emphasise the importance of gelatinous forms in the nekton community. Except for the deepest layer $(800-1000 \mathrm{~m})$ total biomass was one order of magnitude higher in the oceanic Scotia Sea than at the South Georgia slope (Table 2). This difference was mostly due to the large numbers of Salpa thompsoni, Atolla wyvillei and Periphylla periphylla at the oceanic site. In particular, $S$. thompsoni contributed a major share of the biomass at Stn 1 with $87 \%$ in the $0-200 \mathrm{~m}$ layer, and $48 \%$ in the upper $1000 \mathrm{~m}$ during the night collections. The biomass of mesopelagic fish was similar at both stations over $0-1000 \mathrm{~m}$, with $4.92 \mathrm{~g}$ wet wt $\mathrm{m}^{-2}$ at Stn 1 and $4.16 \mathrm{~g}$ wet $w \mathrm{~m}^{-2}$ at Stn 2 (Table 3), but was 
more important in relative terms at the latter where it accounted for $18 \%$ of the biomass, as opposed to $6 \%$ at Stn 1. Euphausiids, notably Euphausia triacantha, were the principal components of the biomass at Stn 2 where they comprised $6 \%$ of the nekton biomass $\left(1.46 \mathrm{~g}\right.$ wet wt $\mathrm{m}^{-2}$ in $\left.0-1000 \mathrm{~m}\right)$. We consider our biomass estimations to be reasonably accurate since, apart from the fast swimming cephalopods, the gelatinous forms, crustaceans and the sluggish mesopelagic fish are readily sampled by the large RMT 25 . Biomass estimates for the highly mobile species such as squid (e.g. Brachioteuthis ?picta with only $75 \mathrm{mg}$ wet wt $\mathrm{m}^{-2}$, $0-1000 \mathrm{~m}$, Stn 2) are likely considerable underestimates since there is evidence to suggest large numbers of squid in the area, particularly ommastrephids (Rodhouse \& Yeatman 1990, Rodhouse 1991). Moreover, squid form the principal dietary component of many top predators in the South Georgia area (e.g. Croxall \& Lishman 1987, Rodhouse et al. 1987, 1990, 1992a, Rodhouse 1991, Rodhouse \& Prince 1993).

The nekton biomass levels for the South Georgia slope region $\left(23.7 \mathrm{~g}\right.$ wet wt $\mathrm{m}^{-2}, 0-1000 \mathrm{~m}$, during the night) are in the same range as others reported for similar micronekton/nekton assemblages in the area. Lancraft et al. (1989) estimated $28.2 \mathrm{~g}$ wet $\mathrm{wt} \mathrm{m}^{-2}$ $(0-1000 \mathrm{~m}$, night tow $)$ for Weddell Sea and $31.0 \mathrm{~g}$ wet wt $\mathrm{m}^{-2}(0-1000 \mathrm{~m}$, night tow $)$ for open waters near ice edge zones in southern Scotia Sea during summer. Even during winter micronekton community biomass has been reported at $22.9 \mathrm{~g}$ wet wt $\mathrm{m}^{-2}(0-1000 \mathrm{~m}$, nighttime) below the partially consolidated pack ice in the vicinity of the Weddell-Scotia Confluence (Lancraft et al. 1991). As in our study, salps, coelenterates and mesopelagic fish were found to contribute the major proportion of the nekton biomass. However, the high biomass levels found in the oceanic Scotia Sea during the present study (Table 2, Stn 1) far exceed all earlier estimations. Our data further suggest a substantial increase in biomass for the surface layers during the night, mostly due to animals migrating from the CDW to the productive upper layers (Table 2, Fig. 4). At the South Georgia slope there is even some evidence for a migration of nekton from below $1000 \mathrm{~m}$ into the upper layers, since total biomass for the upper $1000 \mathrm{~m}$ is increased by more than a factor of 2 during the night (Table 2).

In contrast with previous studies, where Salpa thompsoni was reported to show a weak vertical migration (Piatkowski 1985a, b, Lancraft et al. 1989), this study showed a very marked upward migration during the night (Fig. 4). However, this could also have been due to a patchy distribution, i.e. during the night hauls a particularly dense concentration happened to be sampled in the top $200 \mathrm{~m}$. A patchy distribution pattern could also explain the comparatively dense con- centration of Euphausia superba during a daylight haul in the upper $200 \mathrm{~m}$ at Stn 2 (Fig. 4).

Calycopsis borchgrevinki, Euphausia triacantha and the dominant mesopelagic fish all showed a migration, ascending from the core of the CDW into the surface layers during darkness. For the mesopelagic fish this migration pattern confirms the results of Torres \& Somero (1988). For Electrona antarctica, Gymnoscopelus braueri, Krefftichthys anderssoni and Protomyctophum bolini (4 myctophid species also comprising the key members of the fish biomass in our study) they reported a daytime centre of distribution between 200-600 $\mathrm{m}$ and an upward shift into the upper $200 \mathrm{~m}$ at night. They further conclude that the diel upward movement of fish can result in a displacement of nearly $20 \%$ of the total fish biomass into the upper surface layer at night. This upward shift can be mostly explained as a feeding migration as there is evidence that Antarctic myctophids feed mainly on copepods, euphausiids, amphipods and euphausiid larvae (Rowedder 1979, Hopkins 1985b, Gerasimova 1991, Lancraft et al. 1991), all abundant in this area (Atkinson \& Peck 1988, Atkinson et al. 1990, Ward et al. 1990).

\section{Food web implications and conclusions}

The nekton community described in this study comprises a major component of the Southern Ocean pelagic ecosystem. Its biomass distribution implies a more diverse food web than previously realised, with mesopelagic fish, coelenterates, decapod crustaceans and squid as major links between the zooplankton and top predators. A similar non-krill-dominated Antarctic food chain has been recently suggested for the coastal Weddell Sea (Boysen-Ennen et al. 1991) with the pelagic Antarctic silverfish Pleuragramma antarcticum as the main link between zooplankton and top predators.

Accepting that myctophids, the main group within the mesopelagic fish, feed primarily on small zooplankton such as copepods (e.g. Metridia gerlachei), ostracods (e.g. Conchoecia spp.) and euphausiid larvae (Hopkins 1985b, Lancraft et al. 1991) and that myctophids form a substantial component of the diet of Antarctic top predators (Croxall \& Lishman 1987, Ainley et al. 1991), then they should be regarded as a crucial link within a non-krill-dominated Antarctic food chain. However, the feeding patterns of nekton community members other than myctophids need more study; the diets of decapod crustaceans and coelenterates are not fully documented and a recent study shows that squid, especially the ommastrephid Martialia hyadesi, preys heavily on myctophids (Rodhouse et al. 1992b), which makes trophic interactions within the nekton community relatively complicated. 
We propose the nekton community of the Scotia Sea to be of major importance to the pelagic ecosystem and suggest a food chain with zooplankton-nekton (mesopelagic fish, squid, coelenterates and decapod crustaceans) - top predators as an important alternative to the more conventional Antarctic food chain, where krill is assumed to be consumed directly by the top predators.

Acknowledgements. We thank Captain Chris Elliott, officers and crew of the RRS 'John Biscoe' for their efforts on our behalf and our colleagues during the cruise, particularly Drs Emma Hatfield and Peter Ward for their help in running the RMT system, counting and measuring of nekton specimens. We are grateful to Drs Renate Haass, Hans-Georg Andres, Francesc Pages, Volker Siegel, Ludwig Tiefenbacher, and Prof. Siebrecht van der Spoel for their help in identifying various species of the nekton community. Drs Wilhelm Hagen and Eugene Murphy critically read the manuscript and provided many helpful comments. U.P. thanks the Deutsche Forschungsgemeinschaft and the British Antarctic Survey for supporting his participation in the RRS 'John Biscoe' cruise during austral summer 1991. Financial support was also obtained from the British-German Academic Research Collaboration Programme (ARC) of the British Council and the Deutscher Akademischer Austauschdienst (Grant No. AT 313-ARC-VI-92/65)

\section{LITERATURE CITED}

Adams, N. J., Brown, C. R. (1989). Dietary differentiation and trophic relationships in the sub-Antarctic penguin community at Marion Island. Mar. Ecol. Prog. Ser. 57: 249-258

Adams, N. J., Klages, N. T. (1987). Seasonal variation in the diet of the king penguin (Aptenodytes patagonicus) at sub-antarctic Marion Island. J. Zool., Lond. 212: 303-324

Ainley, D. G., DeMaster, D. P. (1990). The upper trophic levels in polar marine ecosystems. In: Smith, W. O. Jr (ed.) Polar oceanography, Part B: Chemistry, biology, and geology. Academic Press, San Diego, p. 599-630

Ainley, D. G., Fraser, W. R., Smith, W. O. Jr, Hopkins, T. L., Torres, J. J. (1991). The structure of upper pelagic food webs in the Antarctic: effect of phytoplankton distribution. J. mar. Syst. 2: 111-122

Atkinson, A., Peck, J. M. (1988). A summer-winter comparison of zooplankton in the oceanic area around South Georgia. Polar Biol. 8: 463-473

Atkinson, A., Ward, P., Peck, J. M., Murray, A. W. A. (1990). Mesoscale distribution of zooplankton around South Georgia. Deep Sea Res, 37: 1213-1227

Auster, P. J., Griswold, C. A., Youngbluth, M. J., Bailey, T. G. (1992). Aggregations of myctophid fishes with other pelagic fauna. Environ. Biol. Fish. 35: 133-139

Baker, A. de C., Clarke, M. R., Harris, M. J. (1973). The N. I. O. combination net (RMT 1+8) and further developments of rectangular midwater trawls. J. mar. biol. Ass. U.K. 53: $167-184$

Boysen-Ennen, E., Piatkowski, U. (1988). Meso- and macrozooplankton communities in the Weddell Sea, Antarctica. Polar Biol. 9: 17-35

Boysen-Ennen, E., Hagen, W., Hubold, G., Piatkowski, U. (1991). Zooplankton biomass in the ice-covered Weddell Sea, Antarctica. Mar. Biol. 111: 227-235

Cherel, Y., Ridoux, V. (1992). Prey species and nutritive value of food fed during summer to king penguin Aptenodytes patagonica chicks at Possession Bay, Crozet Archipelago. Ibis 134: 118-127

Clarke, A. (1985). Energy flow in the Southern Ocean food web. In: Siegfried, W. R., Condy, P. R., Laws, R. M. (eds.) Antarctic nutrient cycles and food webs. Springer-Verlag. Berlin, p. $573-580$

Clarke, A., Holmes, L. J. (1987). Notes on the biology and distribution of Pasiphaea species from the Southern Ocean. Br. Antarct. Surv. Bull. 74: 17-30

Croxall, J. P., Lishman, G. S. (1987). The food and feeding ecology of penguins. In: Croxall, J. P. (ed.) Seabirds: feeding ecology and role in marine ecosystems. Cambridge University Press, Cambridge, p. 101-133

Everson, I. (1984). Zooplankton. In: Laws, R. M. (ed.) Antarctic ecology, Vol. 2. Academic Press, London, p. 463-490

Field, J. G., Clarke, K. R., Warwick, R. M. (1982). A practical strategy for analysing multispecies distribution patterns, Mar. Ecol. Prog. Ser. 8: 37-52

Filin, A. A., Gorchinsky, K. V., Kiseleva, V. M. (1991). Biomass of myctophids in the Atlantic sector of the Southern Ocean as estimated by acoustic surveys. SC-CAMLR-SSP/7. Commission for the Conservation of Antarctic Marine Living Resources, Hobart, Australia, p. 417-429

Foxton, P. (1966). The distribution and life history of Salpa thompsoni Foxton with observations on a related species Salpa gerlachei. 'Discovery' Rep. 34: 1-116

Gerasimova, O. V. (1991). Feeding and food intake of Electrona carlsbergi (Täning, 1932) Myctophidae, SCCAMLR-SSP/7. Commission for the Conservation of Antarctic Marine Living Resources, Hobart, Australia, p. $411-416$

Gon, O., Heemstra, P. C. (1990). Fishes of the Southern Ocean. J. L. B. Smith Institute of Ichthyology, Grahamstown, South Africa

Harris, M. J. (1969). Acoustic command system. In: Oceanology International '69. B. P. S. Exhibitions Ltd, London

Haury, L. R., McGowan, J. A., Wiebe, P. H. (1978). Patterns and processes in the time-space scale of plankton distributions. In: Steele, J. H. (ed.) Spatial pattern in plankton communities. Plenum Press, New York, p. 277-327

Hellmer, H. H., Bersch, M., Augstein, E., Grabemann, I. (1985). The Southern Ocean. A survey of oceanographic and marine meteorological research work. Ber. Polarforsch. 26: 1-115

Hempel, G. (1985). On the biology of the polar seas especially the Southern Ocean. In: Gray, J. S., Christiansen, M. E. (eds.) Marine biology of polar regions and effects of stress on marine organisms. John Wiley, Chichester, p. 3-34

Hempel, G. (1987). The krill-dominated pelagic system of the Southern Ocean. Environ. Int. 13: 33-36

Hopkins, T. L. (1985a). The zooplankton community of Croker Passage, Antarctic Peninsula. Polar Biol. 4: 161-170

Hopkins, T. L. (1985b). Food web of an Antarctic midwater ecosystem. Mar. Biol. 89: 197-212

Hopkins, T. L., Lancraft, T. M., Torres, J. J., Donnelly, J. (1993). Community structure and trophic ecology of zooplankton in the Scotia Sea marginal ice zone in winter (1988). Deep Sea Res. 40: 81-105

Hopkins, T. L., Torres, J. J. (1988). The zooplankton community in the vicinity of the ice edge, western Weddell Sea, March 1986. Polar Biol. 9: 79-87

Hulley, P. A. (1981). Results of the research cruises of FRV 'Walther Herwig' to South America. LVIII. Family Myctophidae (Osteichthyes, Myctophiformes). Arch. FischWiss. 31: 1-300

Iwasaki, N., Nemoto, T. (1987). Distribution and community 
structure of pelagic shrimps in the Southern Ocean between $150^{\circ} \mathrm{E}$ and $115^{\circ} \mathrm{E}$. Polar Biol. 8: 121-128

Kock, K. H. (1987). Marine consumers: fish and squid. Environ. Int. 13: 37-45

Kock, K. H. (1992). Antarctic fish and fisheries. Cambridge University Press, Cambridge

Kozlov, A. N., Shust, K. V., Zemsky, A. V. (1991). Seasonal and interannual variability in the distribution of Electrona carlsbergi in the Southern Polar Front area (the area to the north of South Georgia is used as an example). SCCAMLR-SSP/7. Commission for the Conservation of Antarctic Marine Living Resources, Hobart, Australia, p. $337-367$

Lancraft, T. M., Hopkins, T. L., Torres, J. J., Donnelly, J. (1991). Oceanic micronektonic/macrozooplanktonic community structure and feeding in ice covered Antarctic waters during the winter (AMERIEZ 1988). Polar Biol. 11: $157-167$

Lancraft, T. M., Torres, J. J., Hopkins, T. L. (1989). Micronekton and macrozooplankton in the open waters near Antarctic ice edge zones (AMERIEZ 1983 and 1986). Polar Biol. 9: 225-233

Larson, R. J. (1986). Pelagic Scyphomedusae (Scyphozoa: Coronatae and Semaeostomeae) of the Southern Ocean. In: Kornicker, L. (ed.) Biology of the Antarctic Seas XVI. Antarct. Res. Ser. 41: 59-165

Larson, R. J., Harbison, G. R. (1990). Medusae from McMurdo Sound, Ross Sea including the descriptions of two new species, Leuckartiara brownei and Benthocodon hyalinus. Polar Biol. 11: 19-25

Mackintosh, N. A. (1934). Distribution of the macroplankton in the Atlantic sector of the Antarctic. 'Discovery' Rep. 9: $65-160$

McGinnis, R. F. (1982). Biogeography of lanternfishes (Myctophidae) south of $30^{\circ} \mathrm{S}$. In: Pawson, D. L. (ed.) Biology of the Antarctic Sea XII. Antarct. Res. Ser. 35: 1-110

Milliman, J. D., Manheim, F. T. (1968). Observations in deepscattering layers off Cape Hatteras, U.S.A. Deep Sea Res. 15: 505-507

Mills, E. L. (1969). The community concept in marine zoology, with comments on continua and instability in some marine communities: a review. J. Fish. Res. Bd Can. 26: 1415-1428

Omori, M., Hamner, W, M. (1982). Patchy distribution of zooplankton: behavior, population assessment and sampling problems. Mar. Biol. 72: 193-200

Piatkowski, U. (1985a). Distribution, abundance and diurnal migration of macrozooplankton in Antarctic surface waters. Meeresforsch. 30: 264-279

Piatkowski, U. (1985b). Maps of the geographical distribution of macrozooplankton in the Atlantic sector of the Southern Ocean. Ber. Polarforsch. 22:1-55

Piatkowski, U. (1989a). Macroplankton communities in Antarctic surface waters: spatial changes related to hydrography. Mar. Ecol. Prog. Ser. 55: 251-259

Piatkowski, U. (1989b). Macrozooplankton communities from Weddell Sea surface waters, Antarctica. Pesq. antárt. bras. 1: $1-10$

Piepenburg, D., Piatkowski, U. (1992). A program for computer-aided analyses of ecological field data. CABIOS 8: $587-590$

Rodhouse, P. G. (1990). Cephalopod fauna of the Scotia Sea at South Georgia: potential for commercial exploitation and possible consequences. In: Kerry, K., Hempel, G. (eds.) Ecological change and the conservation of Antarctic ecosystems. Springer-Verlag, Berlin, p. 289-298

Rodhouse, P. G. (1991). Population structure of Martialia hyadesi (Cephalopoda: Ommastrephidae) at the Antarctic Polar Front and the Patagonian Shelf, South Atlantic. Bull. mar. Sci. 49: 404-418

Rodhouse, P, G., Arnbom, T. R., Fedak, M. A., Yeatman, J., Murray, A. W. A. (1992a). Cephalopod prey of the southern elephant seal, Mirounga leonina L. Can. J. Zool. 70: $1007-1015$

Rodhouse, P. G., Clarke, M. R., Murray, A. W. A. (1987). Cephalopod prey of the wandering albatross Diomedea exulans. Mar. Biol. 96: 1-10

Rodhouse, P. G., Croxall, J. P., Prince, P. A. (1993). Towards an assessment of the stock of the ommastrephid squid Martialia hyadesi in the Scotia Sea: data from predators. In: Okutani, T., O'Dor, R. K., Kubodera, T. (eds.) Recent advances in cephalopod fisheries biology. Tokai University Press, Tokyo, p. 433-440

Rodhouse, P. G., Piatkowski, U., Murphy, E. J., White, M. G., Bone, D. G. (1994). Utility and limits of biomass spectra: the nekton community sampled with the RMT 25 in the Scotia Sea during austral summer. Mar. Ecol. Prog. Ser. 112: $29-39$

Rodhouse, P. G., Prince, P. A. (1993). Cephalopod prey of the black-browed albatross Diomedea melanophrys at South Georgia. Polar Biol. 13: 373-376

Rodhouse, P. G., Prince, P. A., Clarke, M. R., Murray, A. W. A. (1990). Cephalopod prey of the grey-headed albatross Diomedea chrysostoma. Mar. Biol. 104: 353-362

Rodhouse, P. G., White, M. G., Jones, M. R. R. (1992b). Trophic relations of the cephalopod Martialia hyadesi (Teuthoidea: Ommastrephidae) at the Antarctic Polar Front, Scotia Sea. Mar. Biol. 114: 415-421

Rodhouse, P. G., Yeatman, J. (1990). Redescription of Martialia hyadesi Rochbrune and Mabille, 1889 (Mollusca: Cephalopoda) from the Southern Ocean. Bull. Br. Mus. nat. Hist. (Zool.) 56: 135-143

Roe, H. S. J., Baker, A. de C., Carson, R. M., Wild, R., Shale, D. M. (1980). Behaviour of the Institute of Oceanographic Science's rectangular midwater trawls: theoretical aspects and experimental observations. Mar. Biol. 56: 247-259

Romesburg, C. H. (1984). Cluster analysis for researchers. Wadsworth, Belmont

Rowedder, U. (1979). Feeding ecology of the myctophid Electrona antarctica (Günther, 1878) (Teleostei). Meeresforsch. 27: 252-263

Shannon, C. E., Weaver, W. (1963). The mathematical theory of communication. University of Illinois Press, Urbana

Siegel, V., Piatkowski, U. (1990). Variability in the macrozooplankton community off the Antarctic Peninsula. Polar Biol. 10: 373-386

Siegel, V., Skibowski, A., Harm, U. (1992). Community structure of the epipelagic zooplankton community under the sea-ice of the northern Weddell Sea. Polar Biol. 12: 15-24

Stramma, L., Peterson, R. G. (1990). The South Atlantic Current. J. phys. Oceanogr. 20: 846-859

Torres, J. J., Somero, G. N. (1988). Vertical distribution and metabolism in Antarctic mesopelagic fishes. Comp. Biochem. Physiol. 90B: 521-528

Ward, P., Atkinson, A., Peck, J. M., Wood, A. G. (1990). Euphausiid life cycles and distribution around South Georgia. Antarct. Sci. 2: 43-52

Whitworth, T. III (1983). Monitoring the transport of the Antarctic Circumpolar Current at Drake Passage. J. phys. Oceanogr. 13: 2045-2057 